\title{
Asymmetry in Visual Cortical Circuits Underlying Motion-Induced Perceptual Mislocalization
}

\author{
Yu-Xi Fu, Yaosong Shen, Hongfeng Gao, and Yang Dan \\ Division of Neurobiology, Department of Molecular and Cell Biology, University of California, Berkeley, California 94720-3200
}

\begin{abstract}
Motion signals in the visual field can cause strong biases in the perceived positions of stationary objects. Local motion signal within an object induces a shift in the perceived object position in the direction of motion, whereas adaptation to motion stimuli causes a perceptual shift in the opposite direction. The neural mechanisms underlying these illusions are poorly understood. Here we report two novel receptive field (RF) properties in cat primary visual cortex that may account for these motion-position illusions. First, motion signal in a stationary test stimulus causes a displacement of the RF in the direction opposite to motion. Second, motion adaptation induces a shift of the RF in the direction of adaptation. Comparison with human psychophysical measurements under similar conditions indicates that these RF properties can primarily account for the motion-position illusions. Importantly, both RF properties indicate a spatial asymmetry in the synaptic connections from direction-selective cells, and this circuit feature can be predicted by spike-timing-dependent synaptic plasticity, a widespread phenomenon in the nervous system. Thus, motion-induced perceptual mislocalization may be mediated by asymmetric cortical circuits, as a natural consequence of experience-dependent synaptic modification during circuit development.
\end{abstract}

Key words: motion; receptive field; primary visual cortex; asymmetry; STDP; illusion

\section{Introduction}

Perception of object locations in the environment is one of the primary functions of sensory systems, and it involves multiple types of sensory information. In particular, motion signals can exert powerful influences on perceptual localization. In the visual system, the perceived object position is determined not only by the retinal position of the object, but also by movement of the eye (Cai et al., 1997; Ross et al., 1997) and motion of the object itself (Frohlich, 1929; Nijhawan, 1994; Fu et al., 2001). Furthermore, motion signals in the visual field can affect the perceived positions of stationary objects (Ramachandran and Anstis, 1990; De Valois and De Valois, 1991; Snowden, 1998; Nishida and Johnston, 1999; Whitaker et al., 1999; Whitney and Cavanagh, 2000). In one type of motion-induced perceptual mislocalization (here referred to as "type I motion-position illusion"), motion signal within a stationary envelope (e.g., drifting gratings in a stationary Gabor patch) induces a bias in the perceived envelope position in the direction of motion (Ramachandran and Anstis, 1990; De Valois and De Valois, 1991; Whitaker et al., 1999). In another type of motion-position illusion ("type II"), motion adaptation causes not only an illusory movement of a stationary object in the opposite direction (motion aftereffect) (Wohlgemuth, 1911), but also a shift in the perceived object position (Snowden, 1998; Nishida and Johnston, 1999; Whitaker et al.,

Received Nov. 20, 2003; revised Dec. 15, 2003; accepted Jan. 12, 2004.

This work was supported by National Eye Institute Grant R01 EY12561 (Y.D.) and National Natural Science Foundation of China Grant 30300081 (Y.S.). We thank Gerald Westheimer, Marty Banks, Stephen Palmer, and Muming Poo for helpful discussions.

Correspondence should be addressed to Yang Dan at the above address. E-mail: ydan@uclink4.berkeley.edu.

Y. Shen's present address: Institute of Applied Physics and Computational Mathematics, Beijing, 100088, China.

D01:10.1523/JNEUROSCI.5145-03.2004

Copyright $\odot 2004$ Society for Neuroscience $\quad$ 0270-6474/04/242165-07\$15.00/0
1999). Although both type I and type II motion-position illusions are prominent perceptual phenomena, the underlying neural mechanisms remain unknown. As the primary visual cortex (V1) is likely to be important for representing object position at a high resolution, the effects of motion on perceived position are generally assumed to be mediated by the re-entrant connections from motion-processing cortical areas (e.g., MT) to V1 (De Valois and De Valois, 1991; Nishida and Johnston, 1999). Up to now, however, there has been no experimental demonstration of the neural correlates of these illusions.

In this study, we have investigated the mechanisms underlying both type I and type II motion-position illusions. Single-unit recordings from cat primary visual cortex revealed two novel receptive field (RF) properties concerning the interactions between motion and position. First, motion signal within a stationary envelope in the test stimulus causes a displacement of the RF in the opposite direction. Second, adaptation to motion stimuli induces a shift of the RF in the direction of adaptation. Comparison with human psychophysical measurements under similar conditions indicates that these RF properties can largely account for type I and type II motion-position illusions. Furthermore, both RF properties indicate a spatial asymmetry in the synaptic connections from direction-selective cells, a circuit feature that may arise naturally from spike-timing-dependent plasticity of intracortical connections during circuit development.

\section{Materials and Methods}

Visual stimulation and recording. Animal use procedures were as previously described (Yao and Dan, 2001), approved by the Animal Care and Use Committee at the University of California, Berkeley. A total of 18 anesthetized (with sodium pentothal) and paralyzed (with pancuronium bromide) adult cats $(2-3 \mathrm{~kg})$ were used. Single-unit recordings were 
made in area 17 using tungsten electrodes (A-M Systems). Unit isolation was based on cluster analysis of waveforms and the presence of a refractory period determined from the autocorrelograms. Eye movement was minimized by mechanical stabilization. Visual stimuli were generated with a personal computer and presented with a monitor (size: $40 \times 30$ $\mathrm{cm}$; refresh rate: $120 \mathrm{~Hz}$; maximum luminance: $80 \mathrm{~cd} / \mathrm{m}^{2}$; distance from eyes: $114 \mathrm{~cm}$ ). Luminance nonlinearities were corrected through software. For each cell the RF position and size were first estimated by hand mapping, and this information was used to determine the stimulus parameters in computer-controlled mapping. In the experiment shown in Figure 1, the stimulus was a stationary strip (length: $3.8-17.2^{\circ}$; width: $0.24-1.05^{\circ}$ ) containing drifting sinusoidal grating at the preferred orientation of the cell (contrast: 100\%; temporal frequency: $2 \mathrm{~Hz}$; spatial frequency: 3.9 cycles/RF size), which provides salient motion signal within the strip. The background was either dark $\left(<1 \mathrm{~cd} / \mathrm{m}^{2}\right)$ or gray (mean luminance: $40 \mathrm{~cd} / \mathrm{m}^{2}$ ). In each mapping session $(160 \mathrm{sec})$, the strip was presented at 32 positions for each drift direction in a computercontrolled random sequence. Such an interleaved measurement procedure greatly reduces the contribution of slow drifts of the system to the shift in RF position. At each position, the grating was stationary for 450 $\mathrm{msec}$ (at the same spatial phase for the two drift directions), drifting for 2 $\mathrm{sec}$, and stationary again for $50 \mathrm{msec}$, so that the drifting period was separated from stimulus onset and offset by the two stationary periods; spikes were counted only during the drifting period. The spike rate as a function of strip position (Fig. $1 B$ ) was fitted with a Gaussian curve, and the RF position and size were defined as the peak position and the width at half height of the Gaussian fit, respectively. For each cell, 3-11 (mean $=5.2)$ mapping sessions were presented, and the results were averaged. The interval between consecutive sessions was $>1 \mathrm{~min}$. We found that the RF displacements (Fig. $1 B, \Delta X$ ) measured with dark $(9.9 \pm 1.2 \% ; n=96)$ and gray $(9.9 \pm 2.0 \% ; n=31)$ backgrounds were the same ( $p>0.99 ; t$ test), thus the results were combined. In the experiment shown in Figure 2, adaptation was induced with drifting gratings at the optimal orientation of the cell (contrast: 100\%; temporal frequency: $2 \mathrm{~Hz}$; spatial frequency: $\sim 1.5$ cycles/RF size) in an area covering the entire RF. In each mapping block (16 sec), a bar at the optimal orientation (length: $4.9-13.1^{\circ}$; width: $0.17-0.70^{\circ}$ ) was flashed at 32 positions (50 msec/flash, 10 flashes/position) in a computer-controlled random sequence. The bar was either light $\left(80 \mathrm{~cd} / \mathrm{m}^{2}\right.$; background: $\left.<1 \mathrm{~cd} / \mathrm{m}^{2}\right)$ or dark $\left(<1 \mathrm{~cd} / \mathrm{m}^{2}\right.$; background: $\left.80 \mathrm{~cd} / \mathrm{m}^{2}\right)$, depending on which is more effective in driving the cell. Such a stimulus was used for RF mapping to facilitate comparison with the psychophysical experiments (see below). Each experimental session began with two control mapping blocks, followed by $1 \mathrm{~min}$ of adaptation, then by mapping blocks for up to $4 \mathrm{~min}$ to measure the persistence of the adaptation effect. There was no gap between mapping and adaptation. For each cell, such a session was presented alternately for the two directions of adaptation, repeated five times (10 sessions total). The RF position was compared before and after each adaptation block ( $1 \mathrm{~min})$, and the results were averaged across all sessions.

Cell types. In the physiology experiments, all well isolated single units were studied regardless of their laminar locations. However, simple cell RFs (satisfying the criteria in both Hubel and Wiesel, 1962 and Skottun et al., 1991) were poorly mapped with our stimuli (Fig. $1 A$ ), and the segregation between $\mathrm{ON}$ and OFF subregions makes it difficult to determine the overall RF position. Thus only complex cells were included in the analyses. The direction selectivity of these cells (defined as:

$$
\left(R_{\mathrm{p}}-R_{\mathrm{np}}\right) /\left(R_{\mathrm{p}}+R_{\mathrm{np}}\right)
$$

$R_{\mathrm{p}}$ and $R_{\mathrm{np}}$ are responses to full-field drifting gratings at preferred and nonpreferred directions, respectively) ranged from 0 to 0.87 (mean: 0.30 ; SD: $0.22 ; n=127)$. We found no correlation between $\Delta X$ (Fig. $1 B$ ) and the direction selectivity of each cell ( $p>0.5$; ANOVA).

Human psychophysics. Viewing was binocular from $114 \mathrm{~cm}$ with free head. Subjects were instructed to maintain fixation on a cross (size: $0.25^{\circ}$ and $0.33^{\circ}$ for experiments in Figs. 3 and 4, respectively) throughout each session. For the experiment shown in Figure 3, two visual targets were presented in the right half of the visual field, with the vertical gratings inside (contrast: 100\%; temporal frequency: $2 \mathrm{~Hz}$ ) drifting in opposite directions. The spatial frequencies of the gratings were 9.7, 6.7, 5.1, and $4.1 \mathrm{cycles} /{ }^{\circ}$ at the horizontal eccentricities of $2,4,6$, and $8^{\circ}$, respectively, corresponding to four cycles per RF size estimated for human V1 at these eccentricities (see below), matching the physiological experiment shown in Figure 1 (see above). The envelope of each target was a step function (size: $8 \times 8$ cycles of the grating), whose edges were smoothed with a narrow Gaussian filter (width at half height: 1.2 cycles). This filter was used to reduce the perceived horizontal vibration of the visual target at the temporal frequency of the drifting grating, which made it difficult to judge the target position. The filter width, however, was set to be significantly smaller than the estimated human V1 RF size at each eccentricity ( $\sim 30 \%$ RF size) to minimize the effect of target blurring on perceptual mislocalization (Fu et al., 2001). The distance between the two target centers was 10 cycles. In each trial, the targets were presented for $2 \mathrm{sec}$ (same as the physiological experiment), and the subject judged the relative positions of the two targets; a staircase procedure (Gescheider, 1997) was used to measure the perceptual shift (displacement at which the two targets were perceived to be aligned). To reduce systematic bias and the adaptation effect, the grating in each target drifted leftward and rightward equal number of sessions at a random sequence (with the two targets always in opposite directions), and the results from 12 sessions were averaged for each subject (Fig. 3B). For the adaptation experiment (Fig. 4), vertical gratings (contrast: $100 \%$ ) were presented in two rectangular regions $\left(8 \times 2 \%\right.$ region, the center of each region was $2^{\circ}$ from fixation), drifting in opposite directions (temporal frequency: $2 \mathrm{~Hz}$; spatial frequency: $5.9 \mathrm{cycles}^{\circ}{ }^{\circ}$, corresponding to $1.7 \mathrm{cycles} / \mathrm{RF}$ size estimated for human $\mathrm{V} 1$ at $2^{\circ}$ eccentricity). For each test trial (Fig. $4 A$, right panel), two vertical lines $\left(0.052 \pm 1^{\circ} ; 80 \mathrm{~cd} / \mathrm{m}^{2}\right)$ were flashed $(100 \mathrm{msec})$ in the adapted regions, and the subject judged their relative positions. Each session began with a control period, in which two consecutive staircase procedures were used to determine the perceived relative positions of the two lines (the results were averaged). After 1 min of adaptation, perceived line positions were measured again with consecutive staircase procedures over a period of $\sim 2 \mathrm{~min}$. To measure the persistence of the effect, the perceptual shift (difference between perceived positions before and after adaptation) was plotted against the approximate time (within $\sim 10 \mathrm{sec}$ ) at which the staircase procedure was completed (Fig. $4 B$ ). To avoid longterm bias, each subject had the same number of leftward and rightward adaptation sessions at a random sequence, and the results from 12-21 sessions were averaged.

Estimation of human V1 RF size. First we obtained a linear fit of the V1 $\mathrm{RF}$ size as a function of eccentricity measured for the rhesus monkey (Dow et al., 1981) $\left(\right.$ RF size $=0.2^{\circ}+0.07 \times$ eccentricity). Based on the measurement of Spillmann et al. (1987), the sizes of the "perceptive fields" for human and for rhesus monkey can be approximated as $0.9^{\circ}+$ $0.097 \times$ eccentricity and $0.7^{\circ}+0.065 \times$ eccentricity, respectively. Assuming that V1 RF size is linearly proportional to the perceptive field size, we estimated human V1 RF size as a function of eccentricity by scaling both the intercept (by a factor of 0.9/0.7) and the slope (by 0.097/0.065) of the linear fit for the rhesus monkey. The resulting estimate for human V1 RF size can be expressed as $0.26^{\circ}+0.10 \times$ eccentricity. Note that the monkey RF size was measured with the minimum-response-field technique. In our study, however, the RFs were mapped with strips of drifting gratings (Fig. 1) or flashed bars (Fig. 2), and the RF size was defined as the width at half height of the Gaussian fit. When we compared these measurements for the same population of cat primary visual cortical cells ( $n$ $=20$ ), we found that the ratio among these RF size measurements (hand mapping : drifting grating : flashed bar) was 1:0.89:0.64. This ratio was also taken into consideration when we compared the result of each physiological experiment (Figs. 1,2) to the corresponding psychophysical experiment (Figs. 3, 4).

Cortical circuit model. Results shown in Figures 5 and 6 were obtained from simulations of a circuit consisting of 287 neurons, with one postsynaptic target neuron (black, which is not direction-selective, but see Supplemental Data, available at www.jneurosci.org) and two arrays (143 neurons per array) of presynaptic neurons (red and blue). The RFs (size: $2.0^{\circ}$ ) of both arrays were evenly distributed horizontally (distance between neighboring RF centers: $0.03^{\circ}$ ), symmetric with respect to the target RF (same size as presynaptic RFs). Each neuron in the model 
A
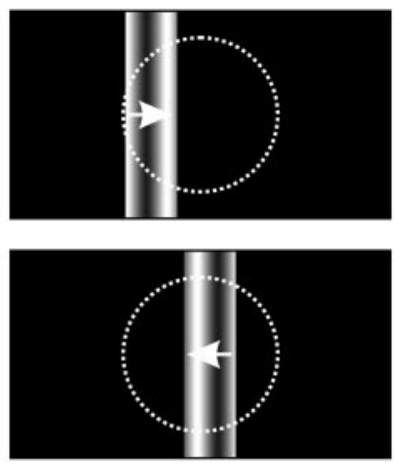

B

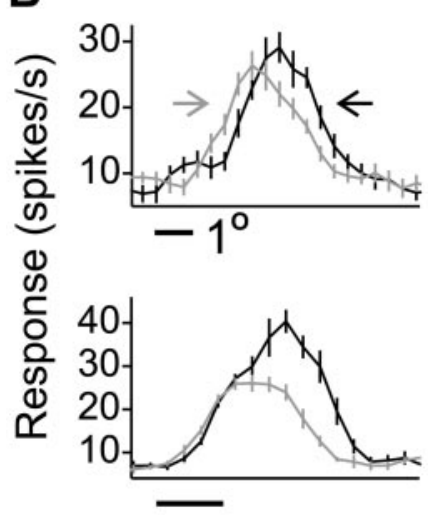

C
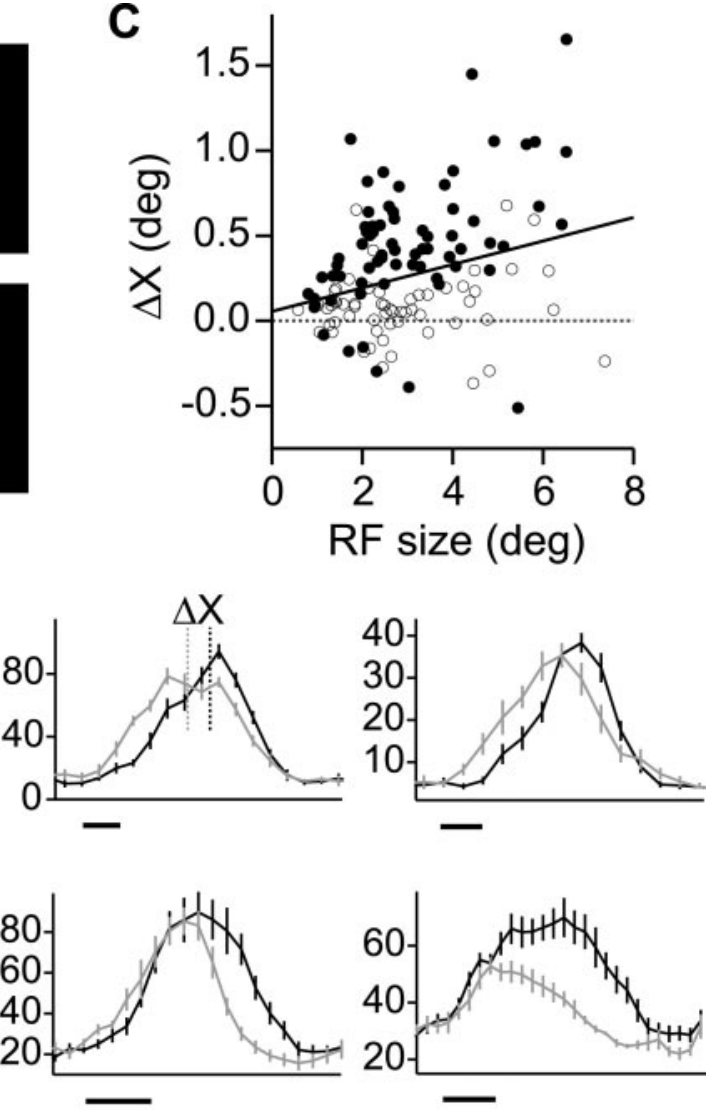

Figure 1. Effect of motion signal on cortical RF position. A, Test stimuli for mapping the RF (see Materials and Methods for stimulus parameters). Arrow indicates direction of motion of the internal grating. The initial phase of the grating was the same for both directions. Dotted circle represents cortical RF. B, RFs of six example neurons recorded in cat visual cortex mapped with the stimuli in $A$, with either rightward (gray line) or leftward (black line) motion. Left is defined as the preferred direction of each cell. Error bar indicates SEM. Dotted vertical line indicates peak position of the Gaussian fit. $\Delta X$, Distance between the gray and black dotted lines. For all six cells $\Delta X$ is significantly different from 0 ( $p<10^{-5}$; nonparametric bootstrap). C, $\Delta X$ versus RF size (width at half height of the Gaussian fit) for all cortical neurons examined ( $n=127)$. Filled circles indicate cells for which $\Delta X$ is significantly different from $0(p<0.05)$. Solid line, Linear fit of the data.

integrated the feedforward and intracortical inputs and generated spikes according to a Poisson process. Details of the model are given in Supplemental Data (available at www.jneurosci.org).

\section{Results}

Motion signal affects cortical RF position

Single-unit recordings were made in the primary visual cortex of anesthetized adult cats. Only complex cells were included in the analysis (see Materials and Methods). We first examined the effect of motion signal in the test stimulus on cortical RFs using stationary strips containing drifting gratings (Fig. $1 A$ ). For each drift direction, the RF was measured by the neuronal response as a function of the strip position. As exemplified in Figure $1 B$, we found a marked effect of motion signal on the measured RF position, with the RF displaced in the direction opposite to grating motion. To quantify this displacement, we fitted each measured RF with a Gaussian function and computed the difference between the peak positions of the Gaussian fits for the two drift directions (Fig. $1 B, \Delta X$ ). The confidence interval for $\Delta \mathrm{X}$ was computed for each neuron from its response variability with nonparametric bootstrap (Efron and Tibshirani, 1993). For the 127 complex cells examined (see Materials and Methods), we found that 63 cells showed significant RF displacement $(p<$
$0.05)$ in the direction opposite to local motion (positive shift, $\Delta X>0$ ), and only six cells showed significant negative shift. At the population level, we found a positive correlation between $\Delta X$ and RF size (Fig. $1 C)(p<0.001$; ANOVA $)$, and the mean displacement ( $\Delta X$ normalized by RF size) was $9.9 \pm 1.0 \%\left(\mathrm{SEM} ; p<10^{-10} ; t\right.$ test $)$. Thus, motion signals in the test stimuli can cause significant displacement of cortical RFs in the opposite direction.

\section{Motion adaptation affects cortical RF position}

We next examined the effect of motion adaptation on cortical RFs. The RF of each neuron was measured with flashed bars before and after adaptation to a highcontrast grating drifting in each of the two directions (Fig. 2A). We found that adaptation for $1 \mathrm{~min}$ induced a shift of the RF in the direction of adaptation (Fig. $2 B$ ). For the total of 56 cells examined, the shift in RF position measured by $\Delta X$ (Fig. $2 B$, sum of the shifts in both directions) was $4.5 \pm$ $1.0 \%$ (SEM; $p<10^{-4} ; t$ test) of the RF size immediately $(<32 \mathrm{sec})$ after adaptation (see Supplemental Data, "Direction selectivity of the target cell," available at www. jneurosci.org). We further examined the persistence of the shift induced by 1 min of adaptation by measuring the RF continuously in the absence of further adaptation, and the effect was found to persist for 1-2 min (Fig. 2C). Thus, motion adaptation can cause a short-term shift of cortical RFs in the direction of adaptation.

\section{Cortical RF properties primarily} account for type I and type II illusions In a typical population-decoding scheme (Deneve et al., 1999), the perceived object position is determined by the spatial profile of the population neuronal response in a retinotopic visual circuit. Assuming that such a scheme is used by the brain to compute object position from the population activity in the primary visual cortex, a shift of the RFs in a given direction should cause a shift of the response profile in the opposite direction, hence a shift in the perceived object position opposite to the RF shift (Fu et al., 2002). Thus, the RF displacement induced by motion (Fig. 1) should cause a perceptual shift in the direction of motion, consistent with type I motion-position illusion (Ramachandran and Anstis, 1990; De Valois and De Valois, 1991; Whitaker et al., 1999), whereas the adaptation-induced RF shift (Fig. 2) should cause a perceptual shift in the opposite direction, consistent with type II motion-position illusion (Snowden, 1998; Nishida and Johnston, 1999; Whitaker et al., 1999). To assess quantitatively the contribution of each motion-related RF property to the corresponding illusion, we measured type I and type II motion-position illusions in human subjects using stimulus parameters matched to those in the physiological experiments and compared the measured effects with those expected from the cortical RF properties.

First, we measured type I illusion in four subjects using visual 

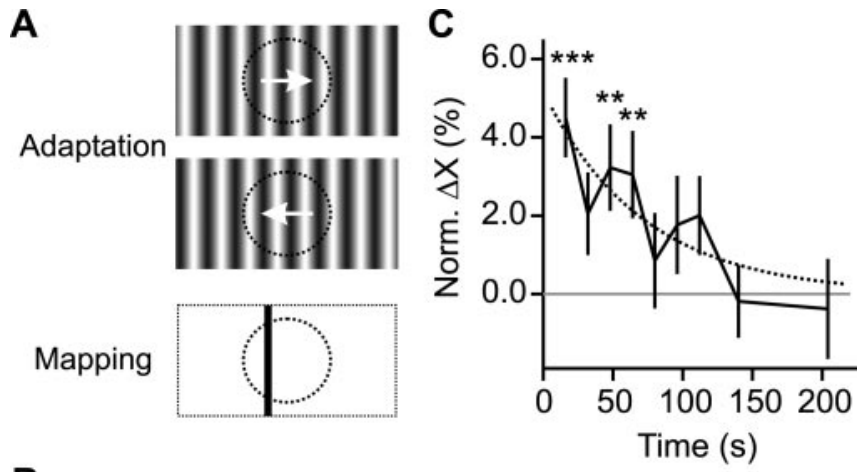

B

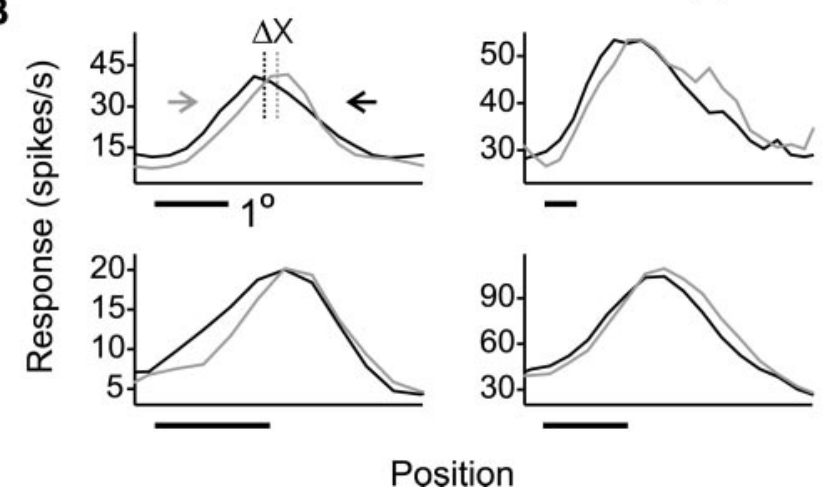

Figure 2. Effect of motion adaptation on RF position. A, Adaptation and mapping stimuli (see Materials and Methods for stimulus parameters). Dotted frame delineating the adapted region was not part of the mapping stimulus. Dotted circle represents cortical RF. B, RFs of four example neurons recorded from cat visual cortex immediately after rightward (gray line) and leftward (black line) adaptation (left: preferred direction of the cell). Control RF mapped before adaptation was omitted for clarity. Dotted vertical line indicates peak position of the Gaussian fit. $\Delta X$, Distance between the gray and the black dotted lines. For all four cells $\Delta X$ is significantly different from 0 ( $p<0.05$; nonparametric bootstrap). Note that because of the response variability and the short persistence of the effect that precludes prolonged mapping, the adaptation-induced RF shift reached $5 \%$ significance only for a small number of cells. However, the effect is highly significant at the population level (see below). C, Persistence of the effect after 1 min of adaptation, measured by $\Delta X$ normalized by RF size for a population of cells. Time 0 is defined as the end of adaptation. Error bar indicates SEM. Asterisks indicate data points significantly different from $0\left({ }^{* *} p<0.01 ;{ }^{* * *} p<0.005 ; t\right.$ test). Dotted line, Exponential fit of the data $\left(\Delta \mathrm{X}_{0} \mathrm{e}^{-\mathrm{t} / \tau}, \Delta \mathrm{X}_{0}=5.2 \%, \tau=72 \mathrm{sec}\right)$.

targets consisting of stationary envelopes and drifting gratings (Fig. 3A). Consistent with previous studies (Ramachandran and Anstis, 1990; De Valois and De Valois, 1991; Whitaker et al., 1999), we found a marked bias of the perceived target position in the direction of grating motion with the magnitude increasing with eccentricity (Fig. 3B). The expected perceptual shift caused by motion-induced RF displacement (Fig. 3B, dashed line) was computed as the measured mean percentage displacement $(9.9 \%$ of RF size, see above) multiplied by the estimated human V1 RF size at each eccentricity (see Materials and Methods). The expected and the measured shifts were found to be in close agreement at all eccentricities tested, indicating that the motioninduced RF displacement can primarily account for type I motion-position illusion.

Next, we measured type II illusion using strips of drifting gratings for adaptation and a pair of flashed bars in the adapted regions to measure perceptual localization (Fig. 4A). Consistent with the previous results (Snowden, 1998; Nishida and Johnston, 1999; Whitaker et al., 1999), we found that 1 min of motion adaptation induced a significant shift in the perceived stimulus position in the opposite direction, which persisted for 1-2 min in the absence of further adaptation (Fig. $4 B$ ). Based on the mea-
A
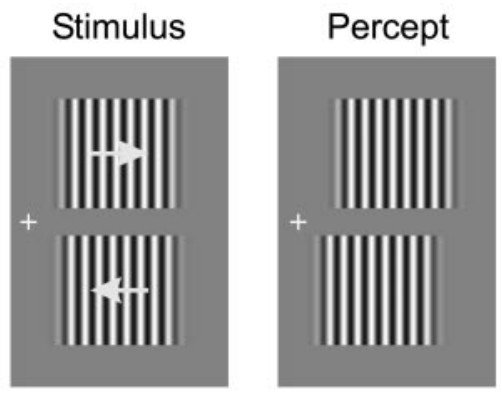

B

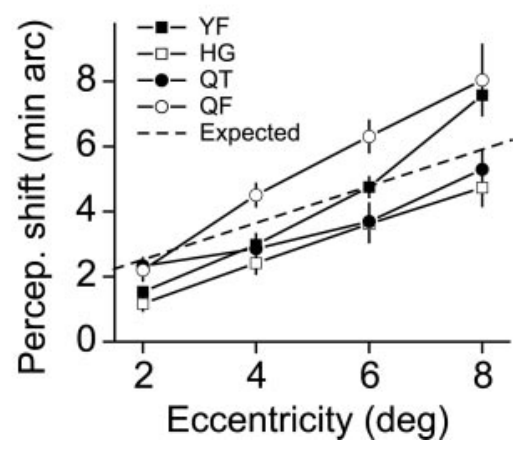

Figure 3. Effect of motion signal on perceived position of stationary object. $A$, Schematic representation of the test stimulus (left panel) and the percept (right panel) in type I motionposition illusion (see Materials and Methods for actual stimulus parameters). In each target the envelope was stationary. Arrow indicates direction of grating motion. Cross, Fixation point. $B$, Perceptual shift as a function of eccentricity measured for two naive subjects (QT, QF) and two authors (YF, HG). Error bar indicates SEM. The effects were significant ( $p<10^{-3} ; t$ test) at all eccentricities for all subjects. Dashed line represents perceptual shift expected from the RF displacement shown in Figure 1.

sured percentage RF shift (Fig. 2C) and the estimated human V1 $\mathrm{RF}$ size at the eccentricity of the test $\left(2^{\circ}\right)$, we computed the expected perceptual effect (Fig. $4 B$, dashed line). The expected and the measured effects were comparable in both the magnitude and time course, indicating that the adaptation-induced RF shift can primarily account for type II motion-position illusion.

\section{Spatial asymmetry in cortical circuits}

What are the mechanisms underlying the effects of motion on RF position? Previous studies have shown that various cortical response properties, including RF size (Sceniak et al., 1999), orientation tuning (Muller et al., 1999; Dragoi et al., 2000), and direction selectivity (Marlin et al., 1988), can be affected by features of the test stimuli and by visual adaptation. These stimulusdependent effects are most likely mediated by synaptic interactions in the cortical circuit rather than by mechanisms intrinsic to the recorded neuron. Here, because the RF position is affected by both motion signal in the test stimuli (Fig. 1) and motion adaptation (Fig. 2), the underlying circuit is likely to involve motionsensitive neurons. In our first observation (Fig. 1), motion signal in each direction affects the two sides of the RF differentially, with rightward (leftward) motion preferentially enhancing the response on the left (right) side of the RF, resulting in a leftward (rightward) RF displacement. This indicates an asymmetry in the spatial distribution of the direction-selective inputs to the cortical neuron, with a leftward (rightward) bias of the excitatory inputs preferring rightward (leftward) motion (Fig. $5 \mathrm{~A}$, middle panel) (see Discussion). Through computer simulations, we found that such an asymmetric circuit robustly reproduced the observed effect of motion on RF position (Fig. $5 A$, bottom panel). In the 
A
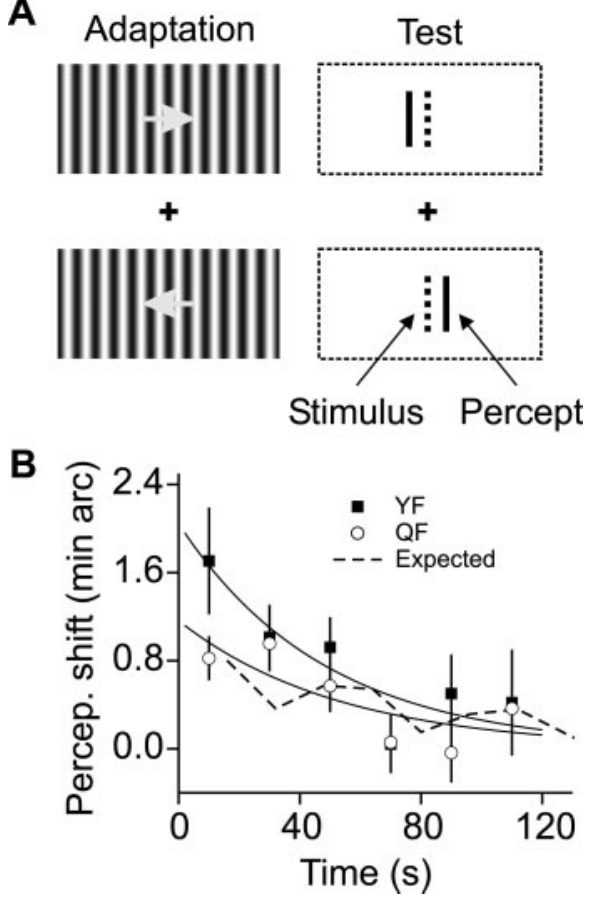

Figure 4. Effect of motion adaptation on perceived object position. A, Schematic representation of the adaptation and test stimuli in type II motion-position illusion (see Materials and Methods for actual stimulus parameters). Arrow indicates the direction of adaptation. Cross, Fixation point. Dotted frames delineating adapted regions were not part of the test stimuli. $B$, Persistence of the perceptual shift after 1 min of adaptation measured for a naive subject (QF) and an author (YF). Time 0 is defined as the end of adaptation. Error bar indicates SEM. Solid line, Exponential fit of the data for each subject (time constants were 48 sec for $Y F$ and 54 sec for QF). Dashed curve represents perceptual shift expected from the RF shift shown in Figure 2.

second observation (Fig. 2), adaptation-induced shift in RF position also requires short-term modification of the inputs in a spatially asymmetric manner. Assuming that adaptation in each direction selectively reduces the excitability of the presynaptic neurons preferring the same direction (Fig. 5B, middle panel), which has been shown for direction-selective neurons in the primary visual cortex (Marlin et al., 1988), RF of the target neuron (black circle) should shift in the direction of adapting motion (Fig. $5 B$, bottom panel), consistent with the experimental observation (Fig. 2). Thus, both effects of motion on RF position observed in the present study support the asymmetric circuit model.

The next question is which neurons provide the directionselective inputs in the asymmetric circuit. Because V1 is the first stage in the geniculocortical pathway where direction selectivity is found, the direction-selective inputs should originate from V1 or higher cortical areas such as MT. Given that the average RF diameter in MT is 10 times that in V1 (Gattass and Gross, 1981), the inputs from MT are less likely to modulate the V1 RF structures at a fine spatial scale. Instead, the direction-selective cells in V1 seem to be the most likely candidates for the presynaptic neurons. As shown below, a spatial asymmetry in the intracortical connections from these cells may arise naturally from spiketiming-dependent plasticity (STDP), a robust phenomenon at the local excitatory connections in the visual cortex (Sjostrom et al., 2001; Froemke and Dan, 2002).

\section{Circuit asymmetry can be predicted by STDP}

In STDP, the direction of synaptic modification depends on the relative timing of presynaptic and postsynaptic spikes: Presynaptic spiking within tens of milliseconds before postsynaptic spiking in-
A
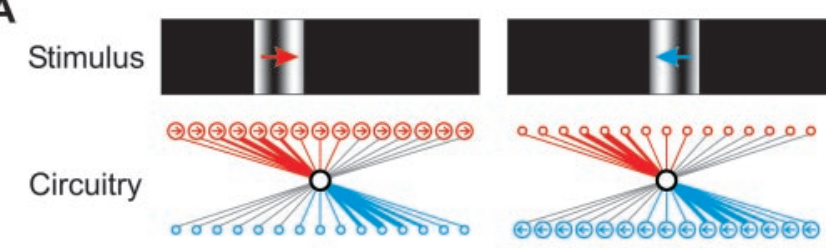
Simulated RF
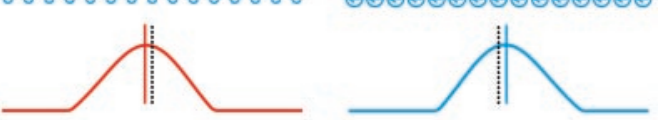

B

Adaptation
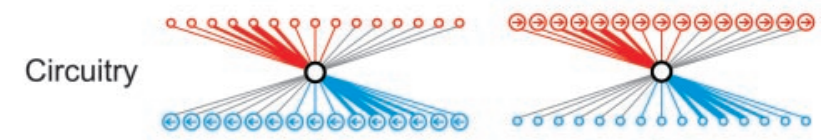
Simulated RF
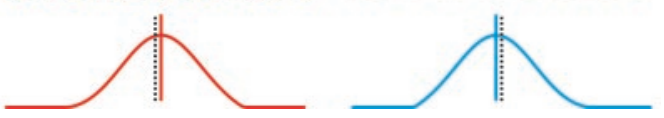

Figure 5. Asymmetric circuit underlying the effects of motion and motion adaptation on RF position. A, Effect of motion signal on RF position in the asymmetric model circuit. Top panel, Stimulus for mapping the RF, same as in Figure 1A. Middle panel, Asymmetric circuit. Red and blue circles represent presynaptic neurons preferring rightward and leftward motion, respectively (arrow indicates preferred direction), and the black circle represents the target neuron. Circle position represents RF center position, and circle size represents the degree of activation evoked by the mapping stimuli (top panel). Line thickness represents connection strength (the weakest connections are shown in gray). Bottom panel, RFs of the target neuron, obtained in a simulation (see Materials and Methods). Solid vertical line (red or blue) indicates RF center position measured with stimuli containing motion signals (top panel), and black dotted line indicates RF position measured without motion signal. $B$, Effect of motion adaptation on RF position. Top panel, Adaptation stimuli (arrow indicates direction of adaptation). Middle panel, Asymmetric circuit. Circle size represents neuronal response to stationary mapping stimuli after adaptation. Bottom panel, Simulated RFs of the target neuron after rightward (red line) and leftward (blue line) adaptation. Dotted black line and solid red/blue line indicate RF center positions before and after adaptation, respectively.

duces synaptic potentiation, whereas spikes in the reverse order result in synaptic depression (Markram et al., 1997; Bi and Poo, 1998). This form of plasticity has been observed widely among excitatory synapses including those in the visual cortex (Sjostrom et al., 2001; Froemke and Dan, 2002). Recent studies have further demonstrated that visual stimuli flashed asynchronously at nearby orientations (Yao and Dan, 2001) or in adjacent regions (Fu et al., 2002) can induce modifications of cortical RFs and human visual perception in a manner consistent with STDP of the intracortical connections (Sjostrom et al., 2001; Froemke and Dan, 2002).

In addition to the flashed stimuli used in the previous studies (Yao and Dan, 2001; Fu et al., 2002), moving objects can also activate neighboring cortical neurons at short temporal intervals and may thus modify their excitatory connections through STDP. Figure $6 A$ depicts a simple model circuit in which the target neuron (black circle) receives excitatory inputs from other neurons preferring rightward (red circles, referred to as " $\rightarrow$ " neurons) or leftward motion (blue circles, “ $\longleftarrow$ " neurons) (see Materials and Methods). By default, the initial distribution of synaptic connections from each group is spatially symmetric. For the " $\rightarrow$ " neurons, a rightward-moving object causes the cells on the left to spike before the target neuron and those on the right to spike afterward (Fig. $6 \mathrm{~B}$, top left plot). Because of STDP, such a spiking pattern strengthens the " $\rightarrow$ " inputs from the left and weakens those from the right. Although leftward motion, which evokes spiking in the reverse order, induces the opposite synaptic mod- 

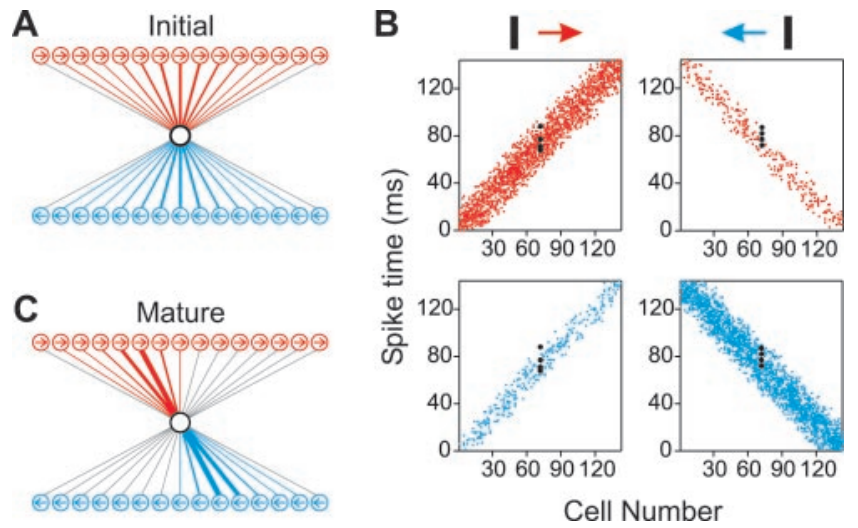

Figure 6. Development of the asymmetric circuit through STDP. A, Initial circuit with symmetric connections. Arrow in each circle represents direction selectivity of the cell. Line thickness represents synaptic strength (the weakest connections are shown in gray). $B$, Simulated spatiotemporal spike patterns in the circuit evoked by a small object (top) moving rightward (left column) or leftward (right column) across the visual field. Each red or blue dot represents a spike of a presynaptic neuron (indexed 1-143, from left to right) with the corresponding color. Black dots represent spikes of the target neuron. C, Mature circuit after extensive exposure to motion stimuli in both directions, during which the connections underwent spike-timingdependent modification. Line thickness is proportional to the synaptic strength after $800 \mathrm{sim}$ ulated motion sweeps in each direction. The velocity of motion in each direction followed a Gaussian distribution (mean: $60 \% \mathrm{sec}$; SD: $20 \% \mathrm{sec}$ ).

ifications, its effect is weaker because the " $\rightarrow$ ” neurons fire fewer spikes (Fig. $6 B$, top right plot). Thus, balanced motion in both directions causes a leftward shift in the distribution of the " $\rightarrow$ " excitatory inputs and, by symmetry, a rightward shift in the " $\longleftarrow$ " inputs (Fig. 6C). Because motion stimuli in various directions are common in the natural environment during development of the visual circuits, the above process should lead to a consistent asymmetry in the adult cortical circuit. As shown above, this asymmetric circuit could account for both motion-related RF properties in adult visual cortex (Fig. 5) and thus form the common basis for type I and type II motion-position illusions.

\section{Discussion}

Visual illusions involving the interaction between motion and perceived position can be divided into two categories. The first category, in which perceptual mislocalization of an object is caused by translational motion of the object itself (Frohlich, 1929; Nijhawan, 1994; Fu et al., 2001), can be explained by mechanisms such as temporal integration (Lappe and Krekelberg, 1998) and "postdiction" (Eagleman and Sejnowski, 2000) in computing the object position or temporally biphasic responses of the visual neurons (Berry et al., 1999; Fu et al., 2001). In the second category, which is mainly represented by type I and type II motion-position illusions (Ramachandran and Anstis, 1990; De Valois and De Valois, 1991; Snowden, 1998; Nishida and Johnston, 1999; Whitaker et al., 1999; also see Whitney and Cavanagh, 2000), the perceptual shifts do not depend on translational motion of the object itself, and thus cannot be explained by the mechanisms described above (Whitney, 2002). Here we have demonstrated two effects of motion on cortical RF position (Figs. $1,2)$ that are ideal candidates for the underlying mechanisms. Because the basic elements of the model, direction-selective cells and spike-timing-dependent plasticity (Fig. 6), are both prevalent in the mammalian visual cortex, the RF properties reported here are most likely to develop in other animals, including humans, and to contribute to the motion-position illusions (Figs. 3, 4). The comparison between the measured perceptual shifts and those expected from the RF properties is, of course, only approximate; it may be affected by factors such as the inaccuracy in the estimated human V1 RF size. Given the prevalence of STDP among excitatory synapses (Markram et al., 1997; Bi and Poo, 1998; Sjostrom et al., 2001; Froemke and Dan, 2002), the spatially asymmetric connections may also develop in other cortical areas containing direction-selective neurons (e.g., MT), which may further contribute to motion-induced perceptual mislocalization. Nevertheless, the close agreement between the expected and the measured perceptual shifts (Figs. $3 B, 4 B$ ) indicates that the RF properties found in the primary visual cortex can primarily, if not completely, account for the two types of motion-position illusions. Interestingly, a recent study of type I motion-position illusion using fMRI revealed a shift in V1 representation in the opposite direction of the perceptual shift (Whitney et al., 2003). Additional studies are required to clarify the relationship between the neuronal spiking responses measured in electrophysiology experiments and the blood oxygen level-dependent signal measured in fMRI.

The neuronal circuit depicted in Figures 5 and 6 provides a simple model for understanding the interactions between motion stimuli and cortical RFs. In this model, we have only considered the excitatory connections. Activity-dependent modification of inhibitory synapses is found to be insensitive to the order of presynaptic and postsynaptic spiking (Woodin et al., 2003), thus motion stimuli should not cause an asymmetry in these connections. Incorporation of symmetric inhibitory connections in the mature circuit does not qualitatively change the effects of motion on target RF position (Fig. 5). We have also assumed that direction-selective cells already exist in the cortex before the development of the asymmetric circuit. Our model does not address the circuitry underlying direction selectivity, although, interestingly, the synaptic connections underlying direction selectivity may also be spatially asymmetric (Livingstone, 1998), the development of which may involve STDP of the intracortical connections (Rao and Sejnowski, 2000) (also see Supplemental Data, available at www.jneurosci.org). Finally, motion stimuli may induce both spike-timing-dependent synaptic modification (Fig. 6) and direction-specific neuronal adaptation (Marlin et al., 1988) (Fig. $5 B$ ), but the two mechanisms were considered separately in the model. Because cortical modification mediated by STDP is relatively long lasting (Markram et al., 1997; Bi and Poo, 1998; Sjostrom et al., 2001; Froemke and Dan, 2002), it allows accumulation of the effect during prolonged circuit development (Fig. 6). On the other hand, contrast adaptation has a transient but strong effect on cortical responses (Marlin et al., 1988), and it is likely to dominate the shortterm effect of motion stimulation (Fig. $5 B$ ). It is also worth noting that, unlike the spatially localized moving objects commonly found in natural scenes, the full-field grating stimuli used in the adaptation experiments (Fig. 2A) should evoke continuous spiking from cortical neurons (especially complex cells) without systematic difference in spike timing between neighboring cells. These stimuli are thus not expected to induce circuit modification through STDP.

In addition to the asymmetric circuit developed through STDP, there are other mechanisms that could mediate the effects of motion on RF position. For example, in a previous study on retinal ganglion cells, a small bar moving across the RF was found to evoke higher responses when entering the RF than when leaving the RF, causing an apparent RF shift in the opposite direction of stimulus motion (Berry et al., 1999). This effect is likely to be mediated by both the transient response property and adaptation of the retinal ganglion cells. These mechanisms, however, cannot account for the motion-induced RF shift found in the present study (Fig. 1). Unlike the moving objects used in the previous study (Berry et al., 1999), our test stimuli consist of stationary 
strips containing local motion signals (Fig. 1A), similar to those used by Barlow and Levick (1965) to study local direction selectivity. Because the strips were presented at different RF positions at a random temporal sequence (see Materials and Methods), the transient response property and the adaptation mechanism should not lead to any systematic RF shift.

Theoretical studies indicate that STDP is a powerful synaptic learning rule (Abbott and Blum, 1996; Gerstner et al., 1996; Roberts, 1999; Kistler and van Hemmen, 2000; Rao and Sejnowski, 2000, 2001; Roberts and Bell, 2000; Song at al., 2000; Song and Abbott, 2001), and its functional implications are under active investigation. In the rat hippocampal CA1 region, STDP of the excitatory connections is believed to mediate asymmetric expansion of neuronal place fields induced by repeated locomotion of the animal (Mehta et al., 2000), which may underlie sequence learning during spatial navigation (Abbott and Blum, 1996). The same study also suggested that STDP can induce asymmetries in visual cortical RFs, which may contribute to direction selectivity (Mehta et al., 2000). In the present study, simulations of a simple model circuit suggest that STDP in the visual cortex allows motion stimuli to induce an asymmetry in the intracortical connections from direction-selective cells, which may account for two novel RF properties, underlying two prominent motion-position illusions. These findings point to a direct causal link between properties of the visual system at the perceptual level and a synaptic learning rule that governs experience-dependent development of the cortical circuits.

\section{References}

Abbott L, Blum K (1996) Functional significance of long-term potentiation for sequence learning and prediction. Cereb Cortex 6:406-416.

Barlow HB, Levick WR (1965) The mechanism of directionally selective units in rabbit's retina. J Physiol (Lond) 178:477-504.

Berry II MJ, Brivanlou IH, Jordan TA, Meister M (1999) Anticipation of moving stimuli by the retina. Nature 398:334-338.

Bi GQ, Poo MM (1998) Synaptic modifications in cultured hippocampal neurons: dependence on spike timing, synaptic strength, and postsynaptic cell type. J Neurosci 18:10464-10472.

Cai RH, Pouget A, Schlag-Rey M, Schlag J (1997) Perceived geometrical relationships affected by eye-movement signals. Nature 386:601-604.

De Valois RL, De Valois KK (1991) Vernier acuity with stationary moving Gabors. Vision Res 31:1619-1626.

Deneve S, Latham PE, Pouget A (1999) Reading population codes: a neural implementation of ideal observers. Nat Neurosci 2:740-745.

Dow BM, Snyder AZ, Vautin RG, Bauer R (1981) Magnification factor and receptive field size in foveal striate cortex of the monkey. Exp Brain Res 44:213-228.

Dragoi V, Sharma J, Sur M (2000) Adaptation-induced plasticity of orientation tuning in adult visual cortex. Neuron 28:287-298.

Eagleman DM, Sejnowski TJ (2000) Motion integration and postdiction in visual awareness. Science 287:2036-2038.

Efron B, Tibshirani RJ (1993) An introduction to the bootstrap. New York: Chapman and Hall.

Froemke RC, Dan Y (2002) Spike-timing-dependent synaptic modification induced by natural spike trains. Nature 416:433-438.

Frohlich FW (1929) Die Empfindungszeit. Jena: Verlag von Gustav Fischer.

Fu YX, Shen Y, Dan Y (2001) Motion-induced perceptual extrapolation of blurred visual targets. J Neurosci 21:RC172.

Fu YX, Djupsund K, Gao H, Hayden B, Shen K, Dan Y (2002) Temporal specificity in the cortical plasticity of visual space representation. Science 296:1999-2003.

Gattass R, Gross CG (1981) Visual topography of striate projection zone (MT) in posterior superior temporal sulcus of the macaque. J Neurophysiol 46:621-638.
Gerstner W, Kempter R, van Hemmen JL, Wagner H (1996) A neuronal learning rule for sub-millisecond temporal coding. Nature 383:76-81.

Gescheider GA (1997) Psychophysics: The Fundamentals, Ed 3. Mahwah, New Jersey: Lawrence Erlbaum Associates, Inc.

Hubel DH, Wiesel TN (1962) Receptive fields, binocular interaction and functional architecture in the cat's visual cortex. J Physiol (Lond) 160:106-154.

Kistler WM, van Hemmen JL (2000) Modeling synaptic plasticity in conjunction with the timing of pre- and postsynaptic action potentials. Neural Comput 12:385-405.

Lappe M, Krekelberg B (1998) The position of moving objects. Perception 27:1437-1449.

Livingstone MS (1998) Mechanisms of direction selectivity in macaque V1. Neuron 20:509-526.

Markram H, Lubke J, Frotscher M, Sakmann B (1997) Regulation of synaptic efficacy by coincidence of postsynaptic APs and EPSPs. Science 275:213-215.

Marlin SG, Hasan SJ, Cynader MS (1988) Direction-selective adaptation in simple and complex cells in cat striate cortex. J Neurophysiol 59:1314-1330.

Mehta MR, Quirk MC, Wilson MA (2000) Experience-dependent asymmetric shape of hippocampal receptive fields. Neuron 25:707-715.

Muller JR, Metha AB, Krauskopf J, Lennie P (1999) Rapid adaptation in visual cortex to the structure of images. Science 285:1405-1408.

Nijhawan R (1994) Motion extrapolation in catching. Nature 370:256-257.

Nishida S, Johnston A (1999) Influence of motion signals on the perceived position of spatial pattern. Nature 397:610-612.

Ramachandran VS, Anstis SM (1990) Illusory displacement of equiluminous kinetic edges. Perception 19:611-616.

Rao RPN, Sejnowski TJ (2000) Predictive sequence learning in recurrent neocortical circuits. In: Advances in neural information processing systems (Solla SA, Leen TK, Muller KR, eds). Cambridge, MA: MIT.

Rao RP, Sejnowski TJ (2001) Spike-timing-dependent Hebbian plasticity as temporal difference learning. Neural Comput 13:2221-2237.

Roberts PD (1999) Computational consequences of temporally asymmetric learning rules: I. Differential Hebbian learning. J Comput Neurosci 7:235-246.

Roberts PD, Bell CC (2000) Computational consequences of temporally asymmetric learning rules: II. Sensory image cancellation. J Comput Neurosci 9:67-83.

Ross J, Morrone MC, Burr DC (1997) Compression of visual space before saccades. Nature 386:598-601.

Sceniak MP, Ringach DL, Hawken MJ, Shapley R (1999) Contrast's effect on spatial summation by macaque V1 neurons. Nat Neurosci 2:733-739.

Sjostrom PJ, Turrigiano GG, Nelson SB (2001) Rate, timing, and cooperativity jointly determine cortical synaptic plasticity. Neuron 32:1149-1164.

Skottun BC, De Valois RL, Grosof DH, Movshon JA, Albrecht DG, Bonds AB (1991) Classifying simple and complex cells on the basis of response modulation. Vision Res 31:1079-1086.

Snowden RJ (1998) Shifts in perceived position following adaptation to visual motion. Curr Biol 8:1343-1345.

Song S, Abbott LF (2001) Cortical development and remapping through spike timing-dependent plasticity. Neuron 32:339-350.

Song S, Miller KD, Abbott LF (2000) Competitive Hebbian learning through spike-timing-dependent synaptic plasticity. Nat Neurosci 3:919-926.

Spillmann L, Ransom-Hogg A, Oehler R (1987) A comparison of perceptive and receptive fields in man and monkey. Hum Neurobiol 6:51-62.

Whitaker D, McGraw PV, Pearson S (1999) Non-veridical size perception of expanding and contracting objects. Vision Res 39:2999-3009.

Whitney D (2002) The influence of visual motion on perceived position. Trends Cogn Sci 6:211-216.

Whitney D, Cavanagh P (2000) Motion distorts visual space: shifting the perceived position of remote stationary objects. Nat Neurosci 3:954-959.

Whitney D, Goltz HC, Thomas CG, Gati JS, Menon RS, Goodale MA (2003) Flexible retinotopy: motion-dependent position coding in the visual cortex. Science 302:878-881.

Wohlgemuth A (1911) On the aftereffect of seen movement. Br J Psychol Monogr Suppl 1:1-117.

Woodin MA, Ganguly K, Poo MM (2003) Coincident pre- and postsynaptic activity modifies GABAergic synapses by postsynaptic changes in $\mathrm{Cl}-$ transporter activity. Neuron 39:807-820.

Yao H, Dan Y (2001) Stimulus timing-dependent plasticity in cortical processing of orientation. Neuron 32:315-323. 